\title{
Projections From the Trigeminal Nuclear Complex to the Cochlear Nuclei: a Retrograde and Anterograde Tracing Study in the Guinea Pig
}

\author{
Jianxun Zhou and Susan Shore ${ }^{\star}$ \\ Department of Otolaryngology and Kresge Hearing Research Institute, University of Michigan, \\ Ann Arbor, Michigan
}

In addition to input from auditory centers, the cochlear nucleus $(\mathrm{CN})$ receives inputs from nonauditory centers, including the trigeminal sensory complex. The detailed anatomy, however, and the functional implications of the nonauditory innervation of the auditory system are not fully understood. We demonstrated previously that the trigeminal ganglion projects to $\mathrm{CN}$, with terminal labeling most dense in the marginal cell area and secondarily in the magnocellular area of the ventral cochlear nucleus (VCN). We continue this line of study by investigating the projection from the spinal trigeminal nucleus to $\mathrm{CN}$ in guinea pig. After injections of the retrograde tracers FluoroGold or biotinylated dextran amine (BDA) in VCN, labeled cells were found in the spinal trigeminal nuclei, most densely in the pars interpolaris and pars caudalis with ipsilateral dominance. The anterograde tracers Fluoro-Ruby or BDA were stereotaxically injected into the spinal trigeminal nucleus. Most labeled puncta were found in the marginal area of VCN and the fusiform cell layer of dorsal cochlear nucleus (DCN). A smaller number of labeled puncta was located in the molecular and deep layers of DCN and the magnocellular area of VCN. The trigeminal projection to CN may provide somatosensory information necessary for pursuing a sound source or for vocal production. These projections may have a role in the generation and modulation of tinnitus.

(c) 2004 Wiley-Liss, Inc.

Key words: somatosensory; pars interpolaris; vocalization; localization; tinnitus

The cochlear nucleus (CN) is a second-order auditory structure that receives inputs from not only other central auditory stations (Shore et al., 1991, 1992) but also from nonauditory structures, including the somatosensory system (Itoh et al., 1987; Wright and Ryugo, 1996; Shore et al., 2000), vestibular system (Gstoettner et al., 1991), and the pontine nuclei (Ohlrogge et al., 2001). These multimodal inputs suggest functional integration between the auditory and nonauditory systems. For example, the cuneate nucleus innervation of cochlear nucleus has been hypothesized to convey information about head and pinna position for the purpose of localizing a sound source in space (Young et al., 1995). In addition, interactions between somatosensory and auditory systems have been linked increasingly to phantom sound perception, also known as tinnitus. This is demonstrated in the observations that injuries of the head and neck region can lead to the onset of tinnitus in patients with no hearing loss (Lockwood et al., 1998).

We demonstrated previously projections from the trigeminal ganglion to $\mathrm{CN}$ in guinea pigs (Shore et al., 2000). Terminal labeling of trigeminal ganglion projections to the $\mathrm{CN}$ was found to be most dense in the marginal cell area and secondarily in the magnocellular area of the ventral cochlear nucleus (VCN). This trigeminal ganglion-cochlear nucleus pathway has been demonstrated to be functionally active (Shore et al., 2003). To date, a projection from the trigeminal nucleus to $\mathrm{CN}$ has not been reported in guinea pigs, although it has been described in cats (Itoh et al., 1987) and rats (Li and Mizuno, 1997) through the use of retrograde tracing studies. Results in both species showed that the projection cells originated in the ipsilateral spinal trigeminal nucleus (Sp5), predominantly in pars interpolaris (Sp5I). There are no studies, however, showing the terminal patterns in $\mathrm{CN}$ arising exclusively from trigeminal nucleus. One study reported termination patterns in cat CN (Itoh et al., 1987) after anterograde tracers were injected into regions span-

Contract grant sponsor: NIH; Contract grant number: R01DC004825, P30 DC05188; Contract grant sponsor: Tinnitus Research Consortium; Contract grant sponsor: The Land Foundation.

${ }^{\star}$ Correspondence to: Susan Shore, Department of Otolaryngology and Kresge Hearing Research Institute, University of Michigan, Ann Arbor, MI 48109. E-mail: sushore@umich.edu

Received 14 July 2004; Revised 3 September 2004; Accepted 8 September 2004

Published online 19 October 2004 in Wiley InterScience (www. interscience.wiley.com). DOI: 10.1002/jnr.20343 
ning both Sp5 and cuneate nucleus. The terminal labeling was found to be in the fusiform cell layer of the dorsal cochlear nucleus (DCN) and granule cell domain of VCN. We investigated the projection from the trigeminal nucleus to the cochlear nucleus in the guinea pig using injections of retrograde tracers into restricted regions of $\mathrm{CN}$ and anterograde tracers restricted to the trigeminal nucleus.

\section{MATERIALS AND METHODS}

Female guinea pigs with normal Preyer's reflexes (292428 g; Elm Hill Breeding Labs, Chelmsford, MA) were used for all experiments. All procedures were carried out in accordance with the NIH guidelines for the care and use of laboratory animals (NIH publication No. 80-23), and guidelines provided by the University of Michigan (UCUCA). Animals were anesthetized with intramuscular injections of ketamine hydrochloride (Ketaset; $80 \mathrm{mg} / \mathrm{kg}$ ) and xylazine (Rompun; $4 \mathrm{mg} / \mathrm{kg}$ ) and placed in a stereotaxic frame (David Kopf, Tujunga, CA). Rectal temperature was monitored and maintained at $38 \pm 0.5^{\circ} \mathrm{C}$ with a thermostatically controlled heating pad. A longitudinal incision was made in the scalp.

\section{Retrograde Experiments}

The left $\mathrm{CN}$ was exposed by removing a small part of occipital bone and aspirating a small portion of the overlying cerebellum. A glass micropipette filled with a solution of $2 \%$ FluoroGold (FG; Fluorochrome, LLC, Denver, CO) $(n=5)$ or 10\% biotinylated dextran-amine (BDA, mol. wt. 10,000 daltons; Molecular Probes, Eugene, OR) $(n=2)$ was placed on the surface of DCN and lowered into the VCN under visual guidance using an operating microscope. For all subjects in the retrograde tracing study, attempts were made specifically to deposit tracers in the lateral region of the posteroventral cochlear nucleus (PVCN), i.e., the small cell cap containing granule cells known to receive somatosensory as well as other nonauditory inputs. Additional injections were made in anteroventral cochlear nucleus (AVCN, $n=2)$ or DCN $(n=1)$. Retrograde tracers $(\sim 0.1 \mu \mathrm{l})$ were injected into VCN using a Hamilton microsyringe equipped with a glass micropipette $(20-30-\mu \mathrm{m}$ tip). After the micropipette was removed, the hole was sealed with dental cement and the skin was sutured. The animal was allowed to recover.

Five to eight days after the injections, animals were euthanized with FetalPlus (Vortech Pharmaceuticals, Dearborn, MI; $5 \mathrm{ml} / \mathrm{kg}$, intraperitoneally [i.p.]) and perfused transcardially with $100 \mathrm{ml}$ of $0.1 \mathrm{M}$ phosphate-buffered saline (PBS; $\mathrm{pH}$ 7.4), followed by $400 \mathrm{ml}$ of $4 \%$ paraformaldehyde in the same buffer. After perfusion-fixation, the brain was isolated and placed in the same fixative for $2 \mathrm{hr}$ at $4^{\circ} \mathrm{C}$. The tissues were transferred into $20 \%$ sucrose in $0.1 \mathrm{M}$ phosphate buffer overnight at $4^{\circ} \mathrm{C}$. The brainstem was then sectioned on a freezing microtome at a thickness of $40-80 \mu \mathrm{m}$. Alternate slides were mounted in serial order on clean glass slides and air dried.

For FG injections, alternate slides were coverslipped with either Gelmount (Biomeda Corp., Foster City, CA) or dehydrated, coverslipped with Micro-cover (Micron, Fairfax, VA), and examined under a fluorescent microscope equipped with a digital camera (Leica DML; Leica Microsystems Wetzlar $\mathrm{GmbH}$, Germany). The second half of the slides was used to reconstruct the labeled cells throughout the brainstem: Slides were counterstained for $1.5 \mathrm{~min}$ in 1\% aqueous neutral red (JT Baker Inc., Phillipsburg, NJ), buffered at pH 4.8 with acetate buffer, then washed in distilled $\mathrm{H}_{2} \mathrm{O}$, dehydrated in graded ethanol, cleared in Micro-clear (Micron), and coverslipped using Micro-cover. For the BDA injection, unless otherwise stated, the process for visualizing BDA products (in both retrograde and anterograde experiments) followed the same procedure as described below. Slides were first incubated for $2 \mathrm{hr}$ in Avidin DH: biotinylated horseradish peroxidase $\mathrm{H}$ complex (Vectastain Elite ABC kit; Vector Laboratories, Burlingame, CA), in $0.1 \mathrm{M}$ phosphate buffer with $0.1 \%$ Triton X-100 (Sigma 9002-93-1), pH 7.4, then reacted with 3,3'-diaminobenzidine tetrahydrochloride (DAB; Vector Laboratories). Slides were counterstained with neutral red, dehydrated, coverslipped, and examined using a light-field microscope (Leica DML).

The brightest injection areas in $\mathrm{CN}$ were determined and represented the injection site. The injection site was found to have a bright core diminishing with a transitional peripheral zone. The brightness diminished to total darkness at the outermost edge. The border of the injection site in $\mathrm{CN}$ was thus determined as the outermost edge of the peripheral zone. Only guinea pigs with injection sites restricted to the confines of the $\mathrm{CN}$ were included in this report $(n=7)$. To reconstruct the injection site after FG injections into $\mathrm{CN}$, photomicrographs of the adjacent counterstained section were digitized and imported into Freehand (Macromedia, Inc.). The CN was drawn on the photomicrograph using the freehand drawing pencil. The photomicrograph of the injection site was digitized, imported to Freehand, and overlapped onto the $\mathrm{CN}$ drawing. Finally, the injection area was outlined and merged onto the layer containing the drawing of $\mathrm{CN}$. In four animals that received single injections aimed at PVCN, the outline of the injection site was separated into subdivisions of DCN and PVCN according to the corresponding overlaps of the injection site with $\mathrm{CN}$, and imported into NIH Image-J along with a reference bar $\left(0.1 \mathrm{~mm}^{2}\right)$ to determine the injection areas in both DCN and PVCN. Nuclear and subnuclear boundaries in trigeminal nucleus were determined with the criteria established in the literature (Jacquin et al., 1986, 1993; Shigenaga et al., 1986). Briefly, the level of the rostral pole of the facial motor nucleus was used as the boundary between the principle nucleus and the spinal trigeminal nucleus (Sp5). The rostral border of pars interpolaris of Sp5 (Sp5I) was defined as the point of the rostral end of the inferior olivary complex, and its caudal border was defined as the point where the "displaced" substantia gelatinosa became entirely contiguous with the spinal tract (Jacquin et al., 1986). The templates were drawn from the counterstained slides at $800-\mu \mathrm{m}$ intervals extending from the oral region to the caudal region of the spinal trigeminal nucleus. Retrogradely labeled neurons in the trigeminal nuclei were visualized using fluorescent microscopy with appropriate filters and mapped directly onto the templates. Retrogradely labeled cells were identified by visualizing a FluoroGold-filled cytoplasm with a central dark area (nucleus). Lower $(5 \times)$ and higher (up to $40 \times$ ) magnifications 
were used frequently to help in mapping and identifying the retrogradely labeled neurons. Photomicrographs of retrogradely labeled neurons were digitized and imported to Adobe Photoshop for labeling and contrast adjustment. Similar reconstruction processes were used for the BDA injections.

\section{Anterograde Experiments}

An opening just lateral-caudal to lambda was drilled in the occipital bone. A glass pipette (tip diameter 20-30 $\mu \mathrm{m}$ ) filled with $10 \% \operatorname{BDA}(n=4)$ or $5 \%$ Fluoro-Ruby (Molecular Probes) $(n=5)$ was lowered into the brain using a micromanipulator. The pipette was positioned into the left spinal trigeminal nucleus according to stereotaxic coordinates $(3 \mathrm{~mm}$ lateral to the midline, $2 \mathrm{~mm}$ caudal to the posterior edge of transverse sinus, and $10.4 \mathrm{~mm}$ ventral to the surface of the dura). Anterograde tracers were either pressure-injected using a Hamilton syringe to a total volume of $0.1 \mu \mathrm{L}$, or iontophoretically delivered by using a constant current generator (Stoelting, $+4 \mu \mathrm{A}$ alternating for up to $20 \mathrm{~min})$. After removing the pipette, the skin was sutured, and the animals were allowed to recover. After 5-6 days, animals were euthanized and perfused transcardially with PBS, followed by $4 \%$ paraformaldehyde in phosphate buffer, $\mathrm{pH} 7.4$. The brain was removed from the skull, postfixed for $2-4 \mathrm{hr}$, and cryoprotected in $20 \%$ sucrose overnight. The tissue sectioning and staining process for Fluoro-Ruby and BDA injections followed the same procedures used for retrograde tracers of FG and BDA, respectively.

Detailed analysis was based on three animals with injection sites restricted to $\mathrm{Sp} 5$ and six animals with injection areas mainly in $\mathrm{Sp} 5$ but with some diffusion into the inferior cerebellar peduncle (icp). The reconstruction procedures for injection sites in $\mathrm{Sp} 5$ were the same as those for $\mathrm{CN}$ in the retrograde experiments. Anterograde labeling in the $\mathrm{CN}$ was examined using bright-field or fluorescent microscopy. Criteria for anterograde projections to the $\mathrm{CN}$ were visualization of both labeled axons, terminals (puncta) on somata, and en passant terminal swellings. Photomicrographs of puncta were digitized and imported to either Adobe Photoshop for labeling and contrast adjustment or to Freehand for the purpose of reconstruction. To obtain clear images of the terminal endings, serial photomicrographs were imported to Metamorph (Universal Imaging Corporation, Downingtown, PA) to generate a Z-projection of stacks. Anterograde terminal endings in the $\mathrm{CN}$ were drawn onto individual transverse slides evenly spanning the $\mathrm{CN}$. Each dot in the drawing represents one or more terminal endings regardless of terminal size or type (i.e., a dot may represent either a large irregular ending or a small bouton).

\section{RESULTS}

\section{Retrograde Experiments}

In four animals that received single injections in the lateral region of PCVN, injection sites were centered in PVCN with some diffusion into the DCN. In the remaining three animals that received more than one injection, the injection sites were centered in either PVCN/AVCN $(n=2)$ or PVCN/DCN $(n=1)$. Deposits of retrograde tracers were confined to the $\mathrm{CN}$ in all seven animals.
Retrogradely labeled cells were found in several areas throughout the brainstem, including the superior olivary complex, trigeminal nuclei, dorsal column nuclei, and reticular formation (Fig. 1). Because our primary interest was the trigeminal projection to the $\mathrm{CN}$, we focused on labeled cells in the trigeminal nuclei. Projection cells were found to be of ipsilateral dominance, and spanned the entire rostral-caudal length of the spinal trigeminal nuclei. The densest concentration of labeled cells was in the pars interpolaris and pars caudalis (Sp5C; Fig. 1 and Table I). The retrogradely labeled neurons were distributed preferentially along the lateral and dorsal column of Sp5, although labeled cells in the ventral column were also observed to a lesser extent. The projection neurons in Sp5 had either polygonal somata, ranging in diameter from $10 \times 12 \mu \mathrm{m}$ to $25 \times 28 \mu \mathrm{m}$, or elongated somata, ranging from $10 \times 30 \mu \mathrm{m}$ to $7 \times 40 \mu \mathrm{m}$ (Fig. $1 \mathrm{~F}, \mathrm{G})$. There were no differences in distribution and morphology of retrogradely labeled neurons in Sp5 between the animals receiving single injections in PVCN and those receiving additional injections in AVCN or DCN. The number of labeled cells in Sp5 was correlated closely with the total injection area in CN (Table I).

\section{Anterograde Experiments}

Each injection site exhibited a densely labeled central core containing numerous intensely labeled neurons. Axons densely filled by tracers were seen originating from the labeled somata in the central core. In three animals, the central core and its surroundings were limited to the trigeminal nuclei without diffusion to the adjacent areas. In the other cases $(n=6)$, injection sites displayed some diffusion to the icp. Results of all these experiments were identical to those with injection sites restricted to the Sp5, and the number of labeled terminal endings in the $\mathrm{CN}$ was related closely to the amount of tracer deposited in the Sp5, not to that in the icp. In addition, five animals with injection sites restricted to lateral or ventral icp showed negative projections to CN (data not shown). We can thus be confident that there was no contamination by "fibers of passage" and that the anterograde projection to $\mathrm{CN}$ in this study originated in the trigeminal nuclei.

Labeled axons emerged from the central core of the injection site, and traveled within the trigeminal spinal tract. Upon entering the $\mathrm{CN}$, fibers followed the dorsal acoustic stria (DAS) or intermediate acoustic stria (IAS), and traveled along the fusiform layer of DCN as well as the marginal area of VCN parallel to the lateral surface of CN (Fig. 2-4). Anterograde projections in $\mathrm{CN}$ were ipsilateral dominant. Labeled puncta were located mostly in the marginal area of VCN and the fusiform cell layer of DCN (Fig. 2-4). A considerable number of puncta, however, was found in the molecular cell layer and deep layers of DCN, as well as the magnocellular regions of AVCN and PVCN (Fig. 4-6). Most labeled fibers were of small-tomedium thickness $(1-2.5 \mu \mathrm{m})$, but a few fibers could be very thin $(\sim 0.4 \mu \mathrm{m})$ (Fig. 4 and 5$)$. 


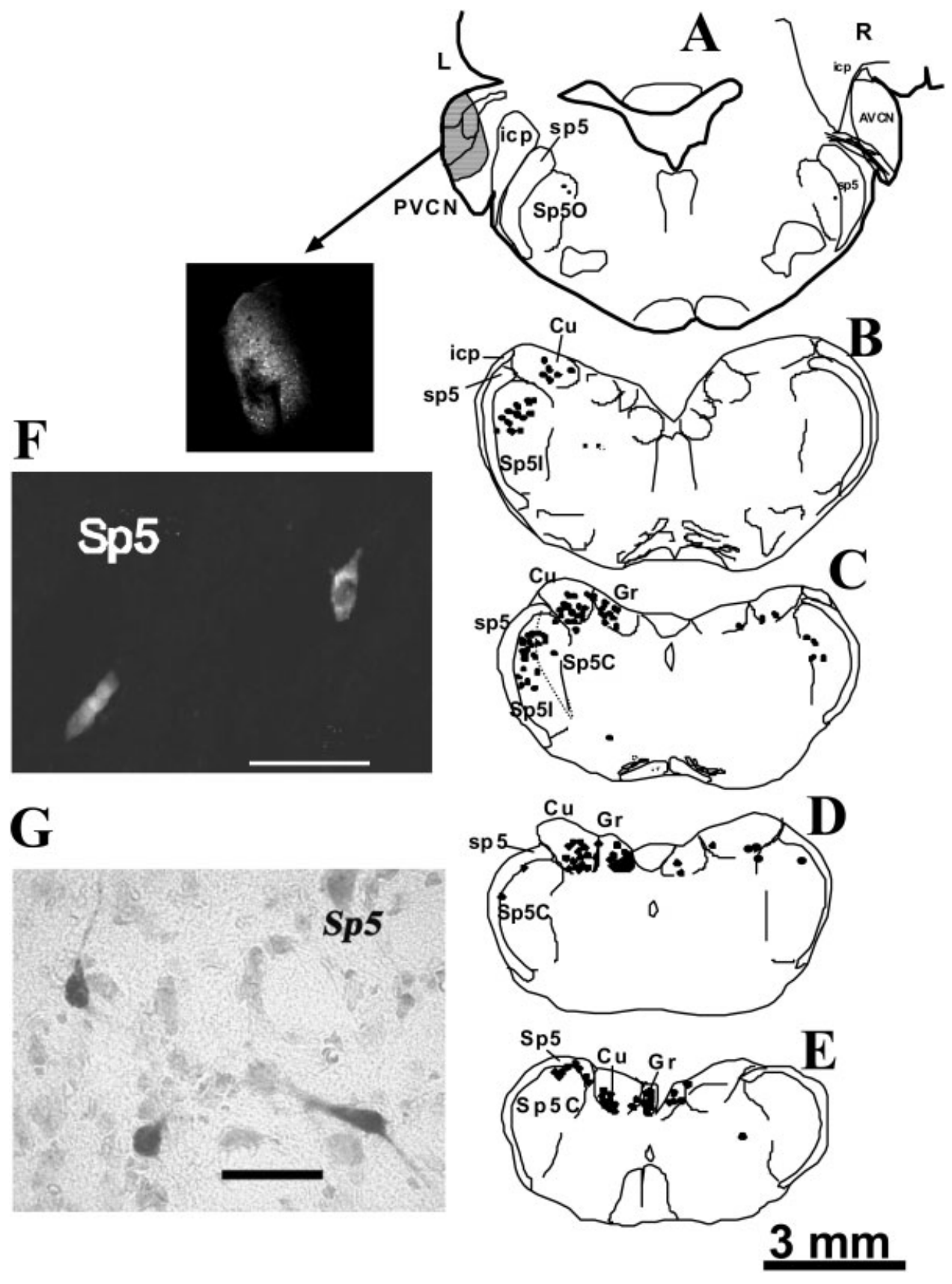

Fig. 1. Retrograde labeling after an injection of FluoroGold (FG) into the cochlear nucleus (CN) in one animal (071603). The shaded area in A represents the injection site in the PVCN. B-E: Drawings of rostral-to-caudal transverse sections of the brain stem. Each drawing represents overlapped serial sections spaced $800 \mu \mathrm{m}$ apart. Each dot represents a labeled neuron. Retrogradely labeled neurons distribute mainly on the ipsilateral side of Sp5, mostly in the pars interpolaris (Sp5I) and pars caudalis (Sp5C). F: Photomicrographs of FG-labeled Sp5 neurons in this animal. G: Photomicrographs of BDA-labeled Sp5 neurons in another animal (062404) after single BDA injection in PVCN. $\mathrm{Sp} 5$, spinal trigeminal nucleus; sp5, spinal trigeminal tract; Sp5I, pars interpolaris of Sp5; Sp5C, pars caudalis of Sp5; icp, inferior cerebellar peduncle; $\mathrm{Cu}, \mathrm{cu}-$ neate nucleus; Gr, gracile nucleus; $\mathrm{CN}$, cochlear nucleus; PVCN, posteroventral cochlear nucleus. Scale bars $=100 \mu \mathrm{m}$ (F); $50 \mu \mathrm{m}(\mathrm{G})$.
Labeled endings seemed to be large irregular terminal swellings giving rise to collaterals, en passant, or small terminal boutons (Fig. 3 and 4). Sometimes several labeled terminal endings crowded to form a cluster (Fig. 3-5). The labeled puncta varied in size, ranging from the largest irregular terminal swelling (diameter of $4-8 \mu \mathrm{m})$ to the smallest bouton type $(0.8-2 \mu \mathrm{m})$. The large, irregular terminal swellings were observed mostly in the marginal areas of VCN and the fusiform cell layer of DCN, whereas the en passant boutons could be found in all subdivisions of CN. Most labeled terminals seemed to make contact primarily with granule cells (Fig. 4 and 5). A few labeled en passant boutons seemed to contact other cell types, however, such as multipolar and bushy cells in VCN as well as giant cells in the deep layer of DCN (Fig. 6A,B).

\section{DISCUSSION}

The present study demonstrates a direct projection from the Sp5 to $\mathrm{CN}$ in the guinea pig. Consistent with earlier retrograde findings in cats (Itoh et al., 1987), the 
TABLE I. Summary of Injection Sites and Cell Counts in Spinal Trigeminal Nuclei After Single Injection of Fluorogold or Biotinylated Dextran Amine in the Posteroventral Cochlear Nucleus`

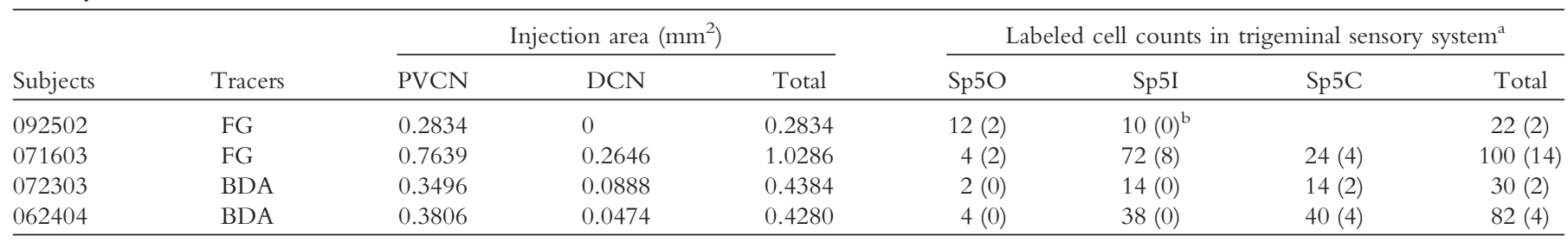

^FG, FluoroGold; BDA, biotinylated dextran amine; PVCN, posteroventral cochlear nucleus; DCN, dorsal cochlear nucleus; Sp5O, pars oralis of spinal trigeminal nucleus; Sp5I, pars interpolaris of spinal trigeminal nucleus; Sp5C, pars caudalis of spinal trigeminal nucleus.

${ }^{a}$ The first number represents cell counts on the ipsilateral side. The number in brackets represents the cell counts on the contralateral side.

${ }^{\mathrm{b}}$ The most caudal end of Sp5I was missed in this subject.

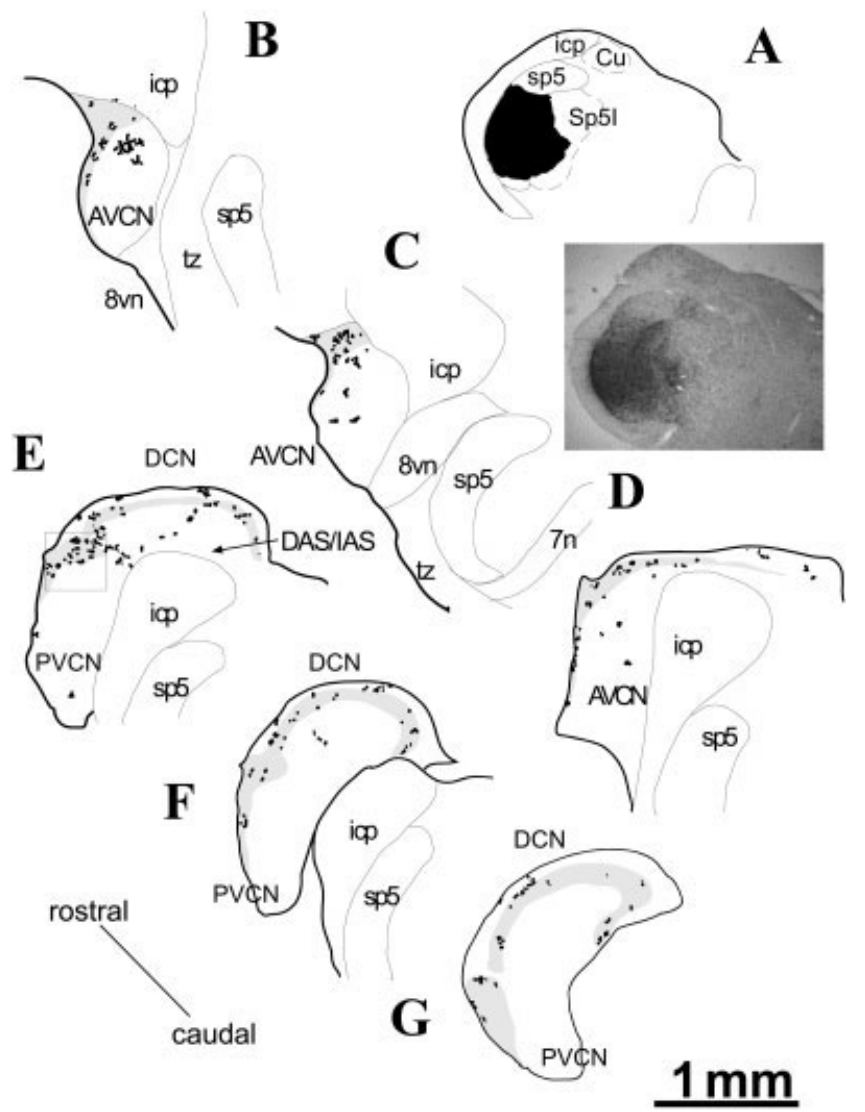

Fig. 2. Reconstruction of anterograde terminal labeling locations in CN. A: BDA injection site in pars interpolaris of Sp5 (Sp5I). B-G: Drawings of transverse sections from the rostral to caudal ends of $\mathrm{CN}$. Each dot represents one or more terminal endings. Marginal area and granular cell layers of $\mathrm{CN}$ are outlined by the shadowed area. Boxed area in $\mathrm{E}$ represents the area of photomicrograph displayed in Figure 3. $\mathrm{CN}$, cochlear nucleus; $\mathrm{Cu}$, cuneate nucleus; AVCN, anteroventral CN; PVCN, posteroventral CN; DCN, dorsal CN; DAS, dorsal acoustic striae; Gr, gracile nucleus; IAS, intermediate acoustic striae; icp, inferior cerebellar peduncle; Sp5, Spinal trigeminal nucleus; sp5, spinal trigeminal tract; Sp5I, pars interpolaris of Sp5; tz, trapezoid body; $7 \mathrm{n}$, facial nerve; $8 \mathrm{vn}$, vestibular nerve. most densely labeled areas within Sp5 after injection of tracers in $\mathrm{CN}$ were $\mathrm{Sp} 5 \mathrm{I}$ and $\mathrm{Sp} 5 \mathrm{C}$. The number of labeled cells was correlated closely with the injection area in $\mathrm{CN}$.

Neurons in Sp5I are classified primarily as lowthreshold mechanoreceptive neurons that respond to nonnoxious tactile stimuli delivered to the head and face, and secondarily as cutaneous nociceptive neurons that respond to noxious mechanical and radiant-heat stimuli (Hayashi et al., 1984). Spinal trigeminal nuclei, including the pars oralis (Sp5O), Sp5I, but especially Sp5C, have been implicated in the mediation of pain (Usunoff et al., 1997). In addition, primary trigeminal afferents responsive to nonnoxious stimuli, such as vibrissa deflection, gentle pressure, tactile stimuli, or jaw movement, innervate all three subdivisions of the Sp5 (Hayashi et al., 1984; Jacquin et al., 1986, 1988, 1993). The retrogradely labeled neurons of varied sizes and shapes observed in the present study likely reflects the heterogeneity of the projection neurons in the Sp5.

Anterograde labeling in the $\mathrm{CN}$ after injections in Sp5 exhibited a wide diversity. There were numerous labeled fibers of small-to-medium size, showing en passant or large irregular terminal swellings that distributed mainly in the marginal area of VCN and fusiform cell layer of DCN. They seemed to contact granule cells. The distribution pattern of the anterograde labeling in the $\mathrm{CN}$ after injection of tracers in Sp5I generally resembled that after injection of tracers in trigeminal ganglion in our previous study (Shore et al., 2000); however, terminal labeling around blood vessels was not observed in $\mathrm{CN}$ in the present study. The terminal distribution pattern also resembled that after injections of tracer in the cuneate nucleus in rats (Wright and Ryugo, 1996), and that after an injection of an anterograde tracer spanning both the spinal trigeminal nucleus and dorsal column nucleus in cats (Itoh et al., 1987). The morphology and distribution of the large, irregular terminal swellings in $\mathrm{CN}$ resemble that of the mossy fibers originating from the cuneate nucleus in rats (Wright and Ryugo, 1996). These mossy fiber inputs to the CN have been hypothesized to provide proprioceptive information related to pinna and head position 


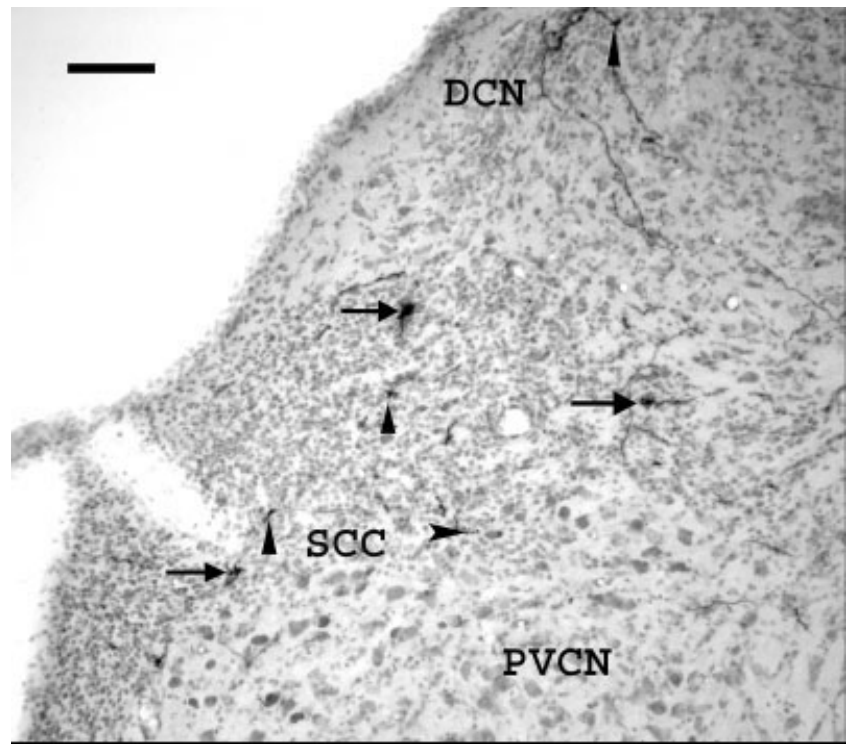

Fig. 3. Photomicrograph $(10 \times)$ of labeled puncta in the marginal area of PVCN (boxed area indicated in Fig. 2E). Terminal labeling was in the form of large irregular swellings (filled triangles), cluster endings (arrows), and en passant (arrowhead). SCC, small cell cap; DCN, dorsal cochlear nucleus; PVCN, posteroventral cochlear nucleus. Scale bar $=100 \mu \mathrm{m}$.

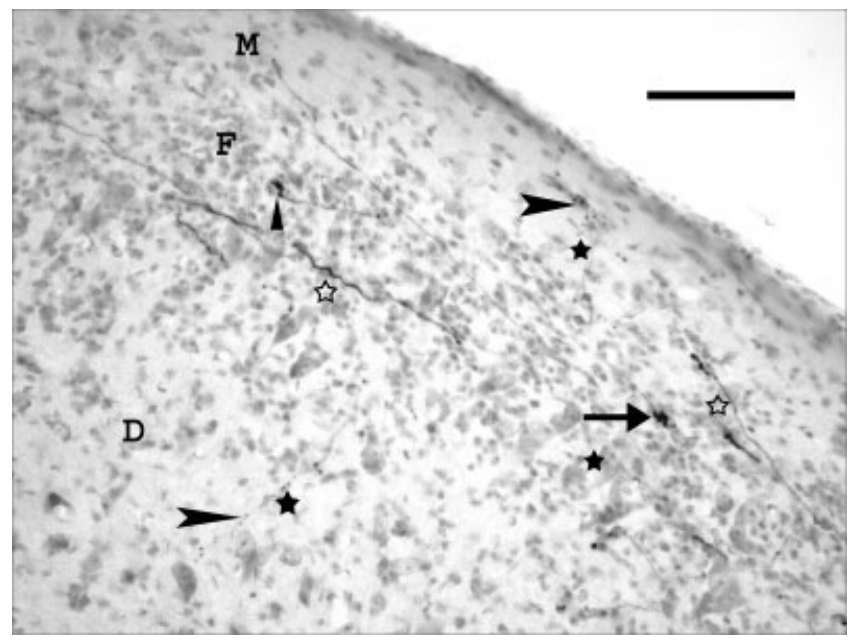

Fig. 4. Photomicrograph $(20 \times)$ of labeled puncta in DCN. Figure was obtained by a Z-projection of stacks of serial photomicrographs in Metamorph. Labeled fibers and puncta were found in all layers of DCN with predominance in the fusiform cell layer. The labeled fibers were of either small-to-medium (hollow stars) or very thin (filled stars). Large irregular swellings (filled triangles) and cluster endings (arrows) were found mostly in fusiform cell layer. Thin fibers and en passant (arrowhead) could be found in all layers of DCN. M, molecular layer; F, fusiform layer; D, deep layer. Scale bar $=100 \mu \mathrm{m}$.

(Young et al., 1995). It has been shown that cells innervating the muscle spindles of the extraocular muscles are located in the trigeminal ganglion (Porter and Donaldson, 1991). It is thus possible that mossy-like fibers from the

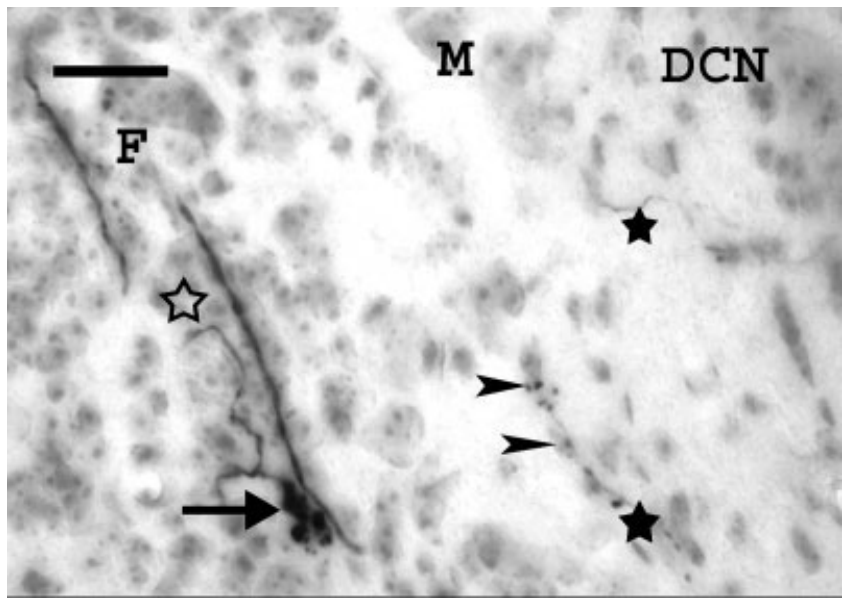

Fig. 5. Higher magnification of labeled puncta in DCN (40×). Figure was obtained by a Z-projection of stacks of serial photomicrographs in Metamorph. Labeled medium-size fibers (hollow stars) in fusiform layer with cluster terminal ending (arrow). Thin fibers (filled stars) in the molecular layer with en passant boutons (arrowheads). M, molecular layer; F, fusiform layer. Scale bar $=25 \mu \mathrm{m}$.
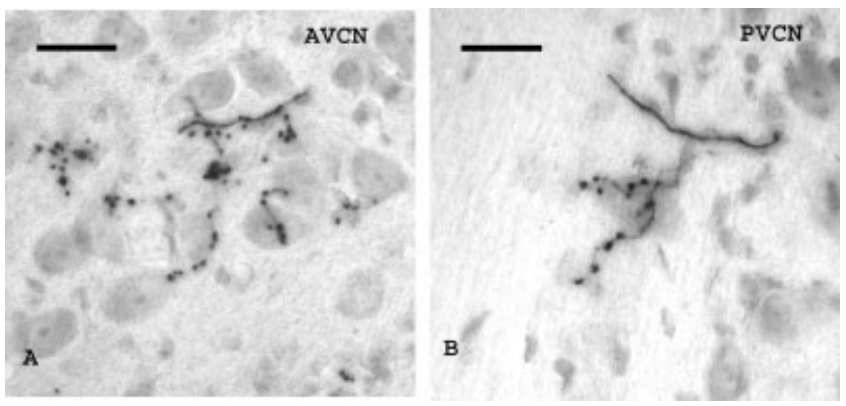

Fig. 6. Photomicrograph $(40 \times)$ of labeled puncta in $\operatorname{AVCN}(\mathbf{A})$ and PVCN $(\mathbf{B})$ after injections of BDA into Sp5. Terminals observed are en passant and also on cell bodies. Scale bars $=25 \mu \mathrm{m}$.

trigeminal system may provide proprioceptive information related to eye-to-head orientation. The two separate somatosensory systems together may provide head-to-space and eye-to-head orientations for the purpose of pursing the sound source, which is important for animals in finding prey or recognizing danger.

Although sound localization may be one function of a somatosensory-auditory interaction, another important purpose of the trigeminal system is to provide information about vocal structures for vocalization. Several trigeminal sensory brainstem subdivisions receive proprioceptive inputs from vocal structures (Romfh et al., 1979; Capra, 1987; Nazruddin et al., 1989; Takemura et al., 1991; Suemune et al., 1992). For example, primary afferent neurons innervating the tongue muscles project to the caudal principal nucleus, Sp5I, and Sp5C in cat (Nazruddin et al., 1989). In addition, the temporomandibular joint and nonencapsulated tongue muscle fibers are innervated 
by cells in the mandibular region of the trigeminal ganglion (Romfh et al., 1979; Capra, 1987; Suemune et al., 1992), which in turn project to Sp5.

In addition to receiving proprioceptive inputs from vocal structures, Sp5 contains premotor neurons that project to trigeminal motor neurons innervating muscles coordinating jaw opening and closing (Li et al., 1995). The present study also indicates that inputs from Sp5 distribute more broadly in the $\mathrm{CN}$ than do those from the cuneate nucleus observed in rats (Wright and Ryugo, 1996). The labeled puncta after injections of tracer in Sp5 were found in the molecular and deep layers of DCN, as well as in AVCN and PVCN. Examination under light microscopy suggests that they make contacts with the principal neurons in these subdivisions. This broader distribution of the Sp5-CN projection, along with the vocal structure innervation of $\mathrm{Sp} 5$, supports the hypothesis that the trigeminal projection to the CN provides proprioceptive information related to vocal tract configurations during vocalizations for the purposes of communication.

Finally, the broad CN distribution from Sp5 also provides anatomic evidence for trigeminally evoked tinnitus ("somatic tinnitus," Levine, 1999). The activity from the trigeminal system can modulate the neural activity in the central auditory system (Shore et al., 2003), and under certain preexisting pathologic conditions, may cause perception of phantom sound.

\section{ACKNOWLEDGMENTS}

We thank H. Bissinger for excellent technical assistance. Portions of this report were presented at the $27^{\text {th }}$ Annual Midwinter Research Meeting of the Association for Research in Otolaryngology, February 22-27, 2004, Daytona Beach, Florida.

\section{REFERENCES}

Capra NF. 1987. Localization and central projections of primary afferent neurons that innervate the temporomandibular joint in cats. Somatosens Res 4:201-213.

Gstoettner W, Burian M, Zundritsch R, Mayr R. 1991. The origin of the vestibulo-cochlear projection in the guinea pig. Neurosci Lett 122:163166.

Hayashi H, Sumino R, Sessle BJ. 1984. Functional organization of trigeminal subnucleus interpolaris: nociceptive and innocuous afferent inputs, projections to thalamus, cerebellum, and spinal cord, and descending modulation from periaqueductal gray. J Neurophysiol 51:890-905.

Itoh K, Kamiya H, Mitani A, Yasui Y, Takada M, Mizuno N. 1987. Direct projections from the dorsal column nuclei and the spinal trigeminal nuclei to the cochlear nuclei in the cat. Brain Res 400:145-150.

Jacquin MF, Renehan WE, Rhoades RW, Panneton WM. 1993. Morphology and topography of identified primary afferents in trigeminal subnuclei principalis and oralis. J Neurophysiol 70:1911-1936.

Jacquin MF, Stennett RA, Renehan WE, Rhoades RW. 1988. Structurefunction relationships in the rat brainstem subnucleus interpolaris: II. Low and high threshold trigeminal primary afferents. J Comp Neurol 267:107130 .

Jacquin MF, Woerner D, Szczepanik AM, Riecker V, Mooney RD, Rhoades RW. 1986. Structure-function relationships in rat brainstem subnucleus interpolaris. I. Vibrissa primary afferents. J Comp Neurol 243:266-279.

Levine R. 1999. Somatic modulation of Tinnitus appears to be a fundamental attribute of tinnitus. In: Hazell JPW, editor. Proceedings of the Sixth International Tinnitus Seminar. London: Tinnitus and Hyperacusis Center, 2001. p 193-197.

Li H, Mizuno N. 1997. Single neurons in the spinal trigeminal and dorsal column nuclei project to both the cochlear nucleus and the inferior colliculus by way of axon collaterals: a fluorescent retrograde doublelabeling study in the rat. Neurosci Res 29:135-142.

Li YQ, Takada M, Kaneko T, Mizuno N. 1995. Premotor neurons for trigeminal motor nucleus neurons innervating the jaw-closing and jawopening muscles: differential distribution in the lower brainstem of the rat. J Comp Neurol 356:563-579.

Lockwood AH, Salvi RJ, Coad ML, Towsley ML, Wack DS, Murphy BW. 1998. The functional neuroanatomy of tinnitus: evidence for limbic system links and neural plasticity. Neurology 50:114-120.

Nazruddin, Suemune S, Shirana Y, Yamauchi K, Shigenaga Y. 1989. The cells of origin of the hypoglossal afferent nerves and central projections in the cat. Brain Res 490:219-235.

Ohlrogge M, Doucet JR, Ryugo DK. 2001. Projections of the pontine nuclei to the cochlear nucleus in rats. J Comp Neurol 436:290-303.

Porter JD, Donaldson IM. 1991. The anatomical substrate for cat extraocular muscle proprioception. Neuroscience 43:473-481.

Romfh JH, Capra NF, Gatipon GB. 1979. Trigeminal nerve and temporomandibular joint of the cat: a horseradish peroxidase study. Exp Neurol 65:99-106.

Shigenaga Y, Okamoto T, Nishimori T, Suemune S, Nasution ID, Chen IC, Tsuru K, Yoshida A, Tabuchi K, Hosoi M, et al. 1986. Oral and facial representation in the trigeminal principal and rostral spinal nuclei of the cat. J Comp Neurol 244:1-18.

Shore S, El-Kashlan HK, Lu J. 2003. Effects of trigeminal ganglion stimulation on unit activity of ventral cochlear nucleus neurons. Neuroscience 119:1085-1101.

Shore SE, Godfrey DA, Helfert RH, Altschuler RA, Bledsoe SC Jr. 1992. Connections between the cochlear nuclei in guinea pig. Hear Res 62: $16-26$.

Shore SE, Helfert RH, Bledsoe SC Jr, Altschuler RA, Godfrey DA. 1991. Descending projections to the guinea pig cochlear nucleus. Hear Res 52:255-268.

Shore S, Vass Z, Wys N, Altschuler R. 2000. The trigeminal ganglion innervates the auditory brainstem. J Comp Neurol 419:271-285.

Suemune S, Nishimori T, Hosoi M, Suzuki Y, Tsuru H, Kawata T, Yamauchi K, Maeda N. 1992. Trigeminal nerve endings of lingual mucosa and musculature of the rat. Brain Res 586:162-165.

Takemura M, Sugimoto T, Shigenaga Y. 1991. Difference in central projection of primary afferents innervating facial and intraoral structures in the rat. Exp Neurol 111:324-331.

Usunoff KG, Marani E, Schoen JH. 1997. The trigeminal system in man. Adv Anat Embryol Cell Biol 136:1-126.

Wright D, Ryugo D. 1996. Mossy fiber projections from the cuneate nucleus to the cochlear nucleus in the rat. J Comp Neurol 365:159-172.

Young ED, Nelken I, Conley RA. 1995. Somatosensory effects on neurons in dorsal cochlear nucleus. J Neurophysiol 73:743-765. 